\title{
Social phobia in spasmodic torticollis
}

\author{
H Gündel, A Wolf, V Xidara, R Busch, A O Ceballos-Baumann
}

\begin{abstract}
Objectives-To study the prevalence of psychiatric comorbidity assessed by the use of a structured clinical interview in a large, representative sample of patients with spasmodic torticollis (ST) and to test the hypothesis that social phobia would be highly prevalent.
\end{abstract}

Methods-In a consecutive cohort of 116 patients with ST treated with botulinum toxin overall psychiatric comorbidity was studied prospectively with the structured clinical interview (SCID) for DSM-IV axis I disorders. Physical disability and psychosocial variables were also assessed with standardised self rating questionnaires.

Results-41.3\% of the subjects met DSM-IV clinical criteria A-G for current social phobia as the primary psychiatric diagnosis. This figure rose to $56 \%$ including secondary and tertiary psychiatric diagnosis. There was no correlation between severity of disease (Tsui score, severity of pain, body image dissatisfaction score) and psychiatric comorbidity. The only significant predictor of psychiatric comorbidity was depressive coping behaviour (logistic regression analysis, $\mathrm{p}<0.01$; $\mathrm{OR}=10.8)$. Compared with a representative sample of the general adult population, in the patients with ST the prevalence of clinically relevant social phobia is 10-fold, of mood disorders 2.4-fold, and of lifetime psychiatric comorbidity 2.6 -fold increased.

Department of

Medicine, Klinikum

rechts der Isar,

Technische Universität

München,

Langerstrasse 3, 81675

München, Germany

$\mathrm{H}$ Gündel

A Wolf

V Xidara

Department of

Neurology

A O Ceballos-Baumann

Institute for Medical

Statistics and

Epidemiology

R Busch

Correspondence to:

Dr H Gündel

H.Guendel@

lrz.tu-muenchen.de

Received 22 September 2000 and in revised form

27 April 2001

Accepted 14 May 2001
Conclusions-A particularly high prevalence of social phobia was found in the cohort of patients with ST. The finding of a high prevalence of social phobia and depressive coping behaviour as the main predictor of psychiatric comorbidity may make a subgroup of patients with ST particularly amenable to specific psychotherapeutic interventions.

\section{(F Neurol Neurosurg Psychiatry 2001;71:499-504)}

Keywords: cervical dystonia; psychiatric comorbidity; social phobia

Spasmodic torticollis (ST), also called cervical dystonia, is a form of focal dystonia in which tonic and phasic involuntary movements of the cervical musculature cause intermittent or sustained deviations of the head and neck. Spasmodic torticollis is the most common form of adult onset focal dystonia. Although $10 \%-20 \%$ of patients may experience remission, nearly all patients relapse within 5 years and are left with persistent disease. ${ }^{1}$
Chronic disease is commonly complicated by psychiatric comorbidity. ${ }^{2}$ Social phobia as significant psychiatric comorbidity coexists with chronic organic conditions such as stuttering and essential tremor ${ }^{3-5}$ which, like ST, compromise social interaction and are exacerbated by stress. However, little is actually known about the nature of the psychiatric comorbidity in ST.

Instead, a fruitless debate as to whether ST is an organic or psychogenic condition has dominated the scientific approach to psychiatric issues in ST. ${ }^{6}$ The disease was once considered "neurotic"7 8 and psychosocially stressful life events before onset of the illness have been found repeatedly in $30 \%-50 \%$ of the patients studied. ${ }^{9-13}$ On the other hand, early reports already described motor abnormalities in one third of the examined group of patients with ST, including $4 \%$ with parkinsonism. ${ }^{14}$ Likewise, Paterson et al suggested that the corpus striatum might be "congenitally weak" and predisposed to torticollis, but at the same time, reported that 16 out of 21 patients with ST in their series had a "shy anxious immature disposition". ${ }^{15}$

In other reports, psychosocial stress in ST has been studied empirically with the aid of standardised questionnaires. ${ }^{9-11}{ }^{16-20}$ Meares reported that patients with ST who remitted had a significant higher score of neuroticism (Eysenck personality inventory) and anxiety (Taylor manifest anxiety scale) than the remainder. This author concluded that this group is amenable to psychiatric treatment. ${ }^{16}$ Matthews et al showed no deviation from the normal in premorbid personality in a group of 30 patients with ST and already drew attention to the severe psychological adverse effects of $\mathrm{ST}$ in that "fear of ridicule and reluctance to leave the home were almost universal". ${ }^{10}$ Nearly $54 \%$ of all patients with ST felt themselves observed and critically looked on by the public and had withdrawn from social activities. ${ }^{17}$

A model of depression in ST was developed according to which the social embarrassment associated with the sense of disfigurement and self depreciation resulting from the abnormal head position leads to social avoidance and depression. ${ }^{112021}$ Indeed, a third of patients were moderately or severely depressed according to Beck depression inventory scores, twice as many as in a control group of patients with cervical spondylosis. ${ }^{20}$ Self depreciation was the most salient predictor for depression in torticollis. $^{21}$

In summary, the picture emerges that increased levels of psychosocial stress in patients with ST are primarily reactive and social withdrawal plays an important part. 
However, psychiatric (DSM-IV) diagnoses were not assigned in previous reports because the subjects were examined primarily with self rating questionnaires. ${ }^{11}{ }^{17-21}$ Therefore, the present study addresses the following question: What is the prevalence of psychiatric comorbidity assessed by the use of a structured clinical interview in a large, representative sample of patients with ST? With special regard to the above cited empirical findings and theoretical models of depression in ST, ${ }^{18-21}$ we hypothesised that social phobia and phobic avoidance would be highly prevalent based on findings in similar conditions such as essential tremor and stuttering.

\section{Method}

PATIENTS

We studied 116 consecutive patients with ST who were referred for botulinum toxin treatment. The interview started shortly after they received their botulinum toxin injections during the same visit. Inclusion criteria for patients with ST were a diagnosis compatible with primary cervical dystonia amenable to botulinum toxin treatment (no other known cause for the dystonia, no tardive dystonia). Additional inclusion criteria were age over 18 years, no history or present use of neuroleptic medication, and ability and willingness to participate in the study. Patients with secondary forms of dystonia, including a positive history of exposure to neuroleptic drugs, and those with other additional focal dystonias more prominent than the ST were excluded. Table 1 gives general sociodemographic information about the patients.

Of the 116 patients, nine $(7.6 \%)$ refused to participate fully in the study and did not fill out the self rating scales. This group of patients did not differ significantly from the group of patients who participated fully in age, sex, duration, or severity (Tsui index) of ST, localised pain, or frequency or effectiveness of treatment with botulinum toxin. All patients gave their informed consent. The study was approved by the local ethics committee.

\section{MEASURES}

Neurological history taking including a standardised questionnaire and physical examinations (including Tsui score ${ }^{22}$ ) were performed by a neurologist who was blind to the results of the clinical psychiatric interview.

Additionally, a version of the structured clinical interview for DSM-IV (SCID-I) was administered. ${ }^{23}{ }^{24}$ The SCID interview covers

Table 1 General sociodemographic characteristics of a group of patients with spasmodic torticollis $(n=116)$

\begin{tabular}{ll}
\hline Age at onset (y): & \\
$\quad$ Mean (SD) & $39.6(14.8)$ \\
Age at initial visit (y) & $51.5(12.6)$ \\
$\quad$ Mean (SD) & \\
Sex: & 53.0 \\
$\quad \%$ Female & 56.0 \\
Education (\%) & 20.7 \\
$\quad$ Basic level & 23.3 \\
$\quad$ Medium level & $11.9(11.3)$ \\
$\quad$ College-preparatory (abitur) & 50.0 \\
$\quad$ Duration of illness (y): & \\
$\quad$ Mean (SD) &
\end{tabular}

most psychiatric diagnoses by specific questions concerning the diagnostic criteria. The reliability and validity of the SCID have been well documented. ${ }^{25}$ According to guidelines concerning interviewer qualifications and training, ${ }^{26}$ the clinically experienced interviewing authors attended a training course for the SCID-I interview technique run by a certified SCID trainer (HUWittchen, Munich), or performed 10 supervised SCID interviews, or both. Patients were assessed blind to the results of the neurological examination. According to DSM-IV guidelines a distinction was made between current (disease manifest in the past 4 weeks) and lifetime (disease not manifest in the past 4 weeks) diagnoses. To qualify for the diagnosis of social phobia in DSM-IV, subjects have to meet criteria A to H. DSM-IV criterion $\mathrm{H}$ specifies that social anxiety may not be in association with a "medical illness factor". The resulting formal assignment of patients with ST meeting DSM-IV criteria A-G, but not $\mathrm{H}$, to the category of anxiety disorder not otherwise specified (300.00), still describes a psychiatric disorder, but it completely blurs the specific clinical situation. As proposed by other authors, ${ }^{3-5}$ we therefore modified DSM-IV criteria to permit a diagnosis of social phobia if only the "clinical" criteria A to G were met. As an indicator of the construct validity of this modified definition of social phobia, we compared social anxiety self ratings among patients with and without the modified social phobia diagnosis.

We compared the prevalence of psychiatric comorbidity in our study group with the current and lifetime prevalence of psychiatric disorders in a representative subsample of the older general population. ${ }^{27}$ This representative sample of the general adult population is described in detail elsewhere. ${ }^{27}$ Briefly, out of a sample ( $n=2524)$ which was randomly drawn from the general population and interviewed by a health research survey company, a smaller simple random, stratified subsample $(n=483)$ was extracted and subjects were interviewed by psychiatrically experienced clinicians (psychiatrists and clinical psychologists) according to DSM criteria. This sample was split according to age as one subsample aged 25-44 years $(n=250)$ and another subsample aged 45-64 years $(n=233)$.

An additional structured interview was developed for the purpose of taking the necessary history. The 41 items covered demographic variables, medical history, the course of the disease, previous treatments, and current neurological symptoms. Items were rated on binary scales, four point rating scales, or VAS scales. Patients assessed their body image dissatisfaction on a self rating VAS scale. Ratings were from 0 to 10 , and higher values indicated greater body image dissatisfaction. 
Symptom checklist-90R

The symptom checklist (SCL-90R) ${ }^{28}{ }^{29}$ is a 90 item questionnaire assessing general psychiatric symptomatology and yields an overall index of psychiatric distress, the general symptomatic index (GSI). The GSI was used in our study to provide a continuous measure of general psychiatric symptoms. Research has documented the reliability and validity of this scale and its German version. ${ }^{29}$

Social phobia scale and social interaction anxiety scale

The social phobia scale (SPS) and social interaction anxiety scale (SIAS) ${ }^{30}$ were used in their validated German versions ${ }^{31}$ to measure two categories of social anxiety. The 20 items of each questionnaire are rated on a five point scale. The literature provides sufficient data on German normal population reference groups. ${ }^{31}$ Higher scores are associated with greater pathology.

\section{Freiburger Fragebogen zur}

Krankheitsverarbeitung

The Freiburger Fragebogen zur Krankheitsverarbeitung (FKV-LIS) ${ }^{32}$ takes into account a wide range of cognitive, emotional, and activity related coping strategies. It consists of 35 items, each rated on a five point scale. The authors describe five factor-analytical scales: depressive coping, active problem focused coping, self distraction, and encouragement, religious faith/search for meaning, and minimisation/wishfulfilling fantasy. Scores indicate the extent of each coping strategy individually used. In depressive coping especially, greater scores are associated with higher pathology. The optimal cut off score was determined for each scale to differentiate high versus low scorers using a receiver operating characteristic (ROC) method.

Life events in the 1 year period before the initial manifestation of ST were assessed with the short version of the Münchner Ereignis Liste (MEL; munich event list). ${ }^{33}$

\section{Results}

Current and lifetime prevalence rates of the axis I psychiatric disorders for ST with patients are presented in table 2 . In each patient, only the clinically most important current psychiatric diagnosis was described and taken into statistical consideration. Analysis shows that of the 116 patients with ST $88(75.9 \%)$ had current psychiatric comorbidity. The most common types were anxiety and mood disorders (together $66.4 \%$ ). Of the 116 patients 58 (50\%; 95\% confidence interval (95\% CI) 40.8-59.2) had a current anxiety disorder.

Using the DSM-IV definition of social phobia but suspending the criterion that prohibits the diagnosis when applied to a patient with a physical disability, we diagnosed 48 (41.3\%; $95 \%$ CI $32.8-51.0)$ of this $58(50 \%)$ patients of the treatment seeking sample as having social phobia as primary psychiatric comorbidity. Considering secondary and tertiary psychiatric diagnosis, 56 (48\%; 95\% CI 39.2-57.6) patients acquired the diagnosis of social phobia. Forty five of the 56 patients $(80 \%$ of this diagnostic sample) with the current diagnosis of social phobia reported that social anxiety had first occurred after the onset of ST - that is, they were reactive.

This assessment/view is supported by the following data: (1) The self rating scale SPS and SIAS showed a higher score ( $t$ test for independent samples: SPS $\mathrm{p}<0.001$; SIAS $\mathrm{p}=0.07$ ) in our study group than in a sample of normal controls. ${ }^{31}$ (2) Self report measures for the patients meeting modified DSM-IV criteria for social phobia were compared with those for the patients who did not fulfill this diagnostic criterion. The patients with an SCID diagnosis of social phobia scored significantly higher (for both scales $\mathrm{p}<0.001$ ) on the two specific measures of social phobia as those without that diagnosis.

The second most frequent current and lifetime diagnoses were mood disorders, usually major depressive disorders (table 2 ). Of the 116 patients, 19 (16.4\%; 95\% CI 10.5-24.6) had a current diagnosis and-additionally to the current diagnosis-62 $(53.4 \% ; 95 \%$ CI 44.0-62.4) a lifetime diagnosis of mood disorder.

Aside from the mentioned anxiety and mood disorders, there were two $(1.7 \%)$ subjects with a current alcohol dependence and nine $(7.6 \%)$ patients with current adjustment disorders. Concerning lifetime diagnosis, nine $(7.8 \%)$ patients had a history of substance dependence, $27(23.3 \%)$ patients had a temporary adjustment disorder, and one $(0.9 \%)$ patient had had an eating disorder.

Table 2 Frequency of psychiatric disorders (DSM-IV) in a group of patients with spasmodic torticollis (ST; $n=116)$

\begin{tabular}{|c|c|c|c|c|}
\hline \multirow[b]{2}{*}{ DSM-IV diagnosis } & \multicolumn{2}{|c|}{ Lifetime } & \multicolumn{2}{|c|}{ Current } \\
\hline & $n$ & $\%$ & $n$ & $\%$ \\
\hline Mood disorders (total) & 62 & 53.4 & 19 & 16.4 \\
\hline Major depressive disorder (with or without melancholic features) (296.2x) & 53 & 45.7 & 15 & 12.9 \\
\hline Dysthymic disorder (300.4) & 3 & 2.6 & 1 & 0.9 \\
\hline Major depressive disorder (bipolar or recurrent) (296.3x) & 9 & 7.8 & 3 & 2.6 \\
\hline Anxiety disorders (total) & 97 & 83.6 & 58 & 50.0 \\
\hline Panic disorder with or without agoraphobia $(300.21 / 300.01)$ & 8 & 6.9 & 3 & 2.6 \\
\hline Agoraphobia without history of panic disorder (300.22) & 9 & 7.8 & 1 & 0.9 \\
\hline Social phobia (300.23) & 82 & 70.7 & 48 & 41.3 \\
\hline Posttraumatic stress disorder (309.81) & 6 & 5.2 & 1 & 0.9 \\
\hline Specific phobia (300.29) & 13 & 11.2 & 5 & 4.3 \\
\hline Alcohol and other substance dependence $(303.90 ; 305.00 ; \ldots)$ & 9 & 7.8 & 2 & 1.7 \\
\hline Eating disorders (307.1) & 1 & 0.8 & - & - \\
\hline Adjustment disorders (309.xx) & 27 & 23.3 & 9 & 7.8 \\
\hline No diagnosis & 10 & 8.6 & 28 & 24.1 \\
\hline
\end{tabular}


Table 3 Prevalence(\%) of the most frequent psychiatric diagnoses in patients with spasmodic torticollis (ST; $n=116)$ compared with the corresponding prevalence in a representative sample of the general population $(n=483)\left(\chi^{2}\right.$ test, $\left.d f=1\right)$

\begin{tabular}{|c|c|c|c|c|c|c|}
\hline ST: $n=116 ;$ controls: $n=483$ & $\begin{array}{l}\text { Current } S T \\
\text { patients }\end{array}$ & $\begin{array}{l}\text { Current } \\
\text { represent. } \\
\text { sample }\end{array}$ & p Value & $\begin{array}{l}\text { Lifetime } S T \\
\text { patients }\end{array}$ & $\begin{array}{l}\text { Lifetime } \\
\text { represent. } \\
\text { sample }\end{array}$ & p Value \\
\hline No diagnosis & 24.1 & 85.4 & 0.000 & 8.6 & 65.0 & $<0.001$ \\
\hline Mood disorders & 16.4 & 6.9 & 0.001 & 53.4 & 13.0 & $<0.001$ \\
\hline Major depressive disorder (296.2/.3) & 15.5 & 3.0 & 0.000 & 52.6 & 9.0 & $<0.001$ \\
\hline Anxiety disorders & 50.0 & 8.1 & 0.000 & 83.6 & 14.0 & $<0.001$ \\
\hline Social phobia $(300.23)$ & 41.4 & 4.1 & 0.000 & 70.7 & 6.5 & $<0.001$ \\
\hline Panic disorder with or without agoraphobia $(300.21 / 300.01)$ & 2.6 & 1.1 & NS & 6.9 & 2.8 & $<0.03$ \\
\hline Other & 6.0 & 0.0 & 0.000 & 45.7 & 2.4 & $<0.001$ \\
\hline
\end{tabular}

There were 43 patients $(37 \%)$ with more than one psychiatric diagnosis. Thirteen patients $(11.2 \%)$ had social phobia and affective disorder.

\section{Comparison with epidemiological findings on} psychiatric morbidity in the general population Relative to the representative sample of the older German general population (range 45-65 years ${ }^{27}$ there were significant differences (table 3). The prevalence of current social phobic syndrome in the ST group was 10-fold that in the general adult population. For current mood disorders the figure was 2.4-fold, and for the lifetime psychiatric comorbidity 2.6-fold. There were no significant differences in age and sex distribution $\left(\chi^{2}\right.$; age $\mathrm{p}=0.19$, sex $\left.\mathrm{p}=0.91\right)$ compared with the representative subsample of the older general population.

Predictors of current psychiatric comorbidity and current social phobia

There was no significant relation between the objective measures of ST, in particular clinical severity (Tsui score) as rated by the neurologist, duration of illness, local pain, frequency of botulinum toxin administration, results of the botulinum toxin treatment, and psychiatric comorbidity (two tailed $\chi^{2}$ or Student's $t$ tests, as appropriate; $\alpha=0.05$ ). To investigate the predictors of current psychiatric comorbidity and current social phobia, a stepwise multiple regression analysis was calculated. Continuous variables were dichotomised by ROC analyses. Objective variables were education, marital status, severity (Tsui score), and extent (isolated pure rotational torticollis versus complex torticollis with adjacent dystonic involvement) of ST, frequency of botulinum toxin administration, duration of the illness $(<5 ; \geqslant 5$ years). Subjective variables were pain (VAS 0-10); extent of psychopathology (SCL-90R mean general symptom index $<0.5$; $\geqslant 0.5)$; incriminating live event within the year before onset of disease yes/no; depressive coping (FKV subscore $<22$; $\geqslant 22$ ); and extent of body image dissatisfaction (visual analogue score $<5 ; \geqslant 5$ ).

The best predictors of current psychiatric comorbidity and social phobia were mostly subjective variables (personal views of the patients, chiefly depressive coping).

Depressive coping was the main predictor of current psychiatric comorbidity $(p<0.01$; odds ratio $=10.8,95 \%$ CI 3.0-40.1) and current social phobia $(\mathrm{p}<0$ 0.01; odds ratio=5.6, 95\% CI 2.2-14.2). Other independent explanatory variables were for the prevalence of current psychiatric comorbidity, an incriminating life event in the 1 year period before the initial manifestation of ST $(\mathrm{p}<0.05$; odds ratio $=6.1,95 \%$ CI 1.3-30.0), and for the diagnosis of social phobia the extent of body image dissatisfaction $(p=0.05$; odds ratio $=2.4,95 \%$ CI 1.0-6.3).

\section{Discussion}

Our study on the prevalence of psychiatric comorbidity yielded two new findings. Firstly, over $75 \%$ of patients with ST had a psychiatric diagnosis when the study was conducted. Secondly, as predicted we found that more than $50 \%$ of the patients with ST fulfilled modified DSM-IV criteria for social phobia. Furthermore, there was a significant correlation between psychiatric comorbidity in accordance with the DSM-IV criteria and a depressive, maladaptive strategy for coping with ST, but not with an objective somatic variable indicating the severity of the disorder (Tsui score).

As a limitation of this study, only some potentially important psychosocial factors were measured in our sample. Thus, a more exhaustive list of psychosocial factors, including social support, personality, locus of control, and other personal outlook variables, may have disclosed an even more substantial association with psychiatric comorbidity.

The level $(75.9 \%)$ of current psychiatric comorbidity in our patients with ST is considerably higher than the values that have been reported world wide in epidemiological studies on psychiatric morbidity in the general population. ${ }^{27}{ }^{34}$. Here, the overall prevalence of psychiatric morbidity is between $29 \%$ and $34 \% .{ }^{27}$ Even compared with the known high levels of psychiatric comorbidity seen in patients with chronic neurological disordersfor example, epilepsy and pseudoepilepsy (psychiatric comorbidity $65 \%$ ) or migraine (psychiatric comorbidity 65\%)-the level in the patients with ST seems high. ${ }^{35} 36$ Our most important finding is the high prevalence of social phobia in ST when this DSM-IV diagnosis was modified to permit a diagnosis of social phobia in patients with disfiguring or disabling physical conditions. ${ }^{3-5}$ In a recent short report ${ }^{37}$ on 44 consecutive patients with ST the authors reported current anxiety disorders in $18 \%(n=8)$, but did not comment on social phobia. The difference between our finding and that of this short report is unclear. Unfortunately, no information was provided on clinical details (for example, severity, treatment, duration of illness), definitions, and 
the training status of the raters who administered the SCID interview. ${ }^{26}$ The construct validity of our classification was supported by the finding that symptoms of social phobia as measured with the two rating scales (SPS, SIAS) assessing social anxiety were significantly worse among patients with the modified social phobia diagnosis in the SCID than in those without this diagnosis. Social phobia, as defined by DSM-IV, is a pattern of excessive fear of scrutiny or embarrassment suffered by persons facing participation in seemingly conventional social interactions. Social phobia is a common affliction, with a point prevalence in the range of $5 \%-7 \%$ in the community. ${ }^{38} 39$ DSM-IV excludes persons with social anxiety secondary to disfiguring or disabling physical conditions from the diagnostic category of social phobia. This exclusion is without empirical basis..$^{3-5}$ Low self esteem based on disturbed body image has been described as the cornerstone of self related negative cognitions in ST. ${ }^{20}$ In patients with other stigmatising physical disabilities such as adult stutterers $^{45}$ and essential tremor, ${ }^{5}$ social anxiety was already identified as an important source of role impairment. ${ }^{4}$ So, if there is a subgroup of patients with ST who experience excessive social anxiety, then it seems most judicious to apply the diagnosis of social phobia to these persons. Beyond the scope of ST, the literature suggests that secondary social anxiety in response to physical conditions is a larger problem than might be expected. ${ }^{3}$ Stein et al found social phobia of clinical significance in $12(75 \%)$ of 16 adult stutterers seeking treatment ${ }^{4}$ and in seven (29\%) of 24 patients with Parkinson's disease. ${ }^{40}$ Thus, prohibiting the diagnosis of social phobia for patients with physical disabilities may hinder the access of these persons to treatment. ${ }^{4}$

Although limited in its scope, this study provides evidence to suggest that the extent of psychiatric comorbidity in our study population did correlate with the depressive, maladaptive attitude with which the patients viewed their illness, and not with the objective severity of ST. A low correlation between the objective severity of an illness with a chronic course and psychosocial stress has been reported in general $^{41}$ and for specific disorders (for example, Parkinson's disease ${ }^{40}$ low back pain, ${ }^{42}$ coronary artery disease ${ }^{43}$ ). For the dystonias, however, it has not yet been described with a structured psychiatric assessment technique. This low correlation may imply that once developed, psychiatric problems often become dissociated from the real extent of physical features in ST but represent a more habitual, dysfunctional attitude towards the illness. Therefore, at least in this subgroup, direct treatment of the psychiatric problems is needed additionally to medical treatment of ST.

More generally, considering the major influence of maladaptive coping style on psychiatric comorbidity in ST, a low threshold, problem orientated, and cost effective training programme for learning to cope with the disorder is the practical management consequence of our results. There are already brief and effective treatment programmes for social phobia, and with little effort they could be adapted to meet the specific needs of patients with ST.

The study was supported by a grant (KKF-Nr 767187) from the TU München.

1 Dauer WT, Burke RE, Greene P, et al. Current concepts on the clinical features, aetiology and management of diopathic cervical dystonia. Brain 1998;12:547-60.

2 Cohen-Cole SH, Brown FW, McDaniel JS. Assessment of depression and grief reactions in the medically ill. In: Stoudemire A, Fogel BS, eds. Psychiatric care of the medical patient. New York: Oxford University Press, 1993:53-69.

3 Oberlander EI, Schneier FR, Liebowitz MR. Physical disability and social phobia. $\mathcal{f}$ Clin Psychopharmacol 1994;14:136- 43 .

4 Stein MB, Baird A, Walker JR. Social phobia in adults with stuttering. Am f Psychiatry 1996;153:278-80.

5 George MS, Lydiard RB. Social phobia secondary to physical disability. A review of benign essential tremor (BET) cal disability. A review of benign essential trem
and suttering. Psychosomatics 1994;35:520-23.

6 Lees AJ. Tics and related disorders. Edinburgh: Churchill LivLees AJ. Tics and related
ingstone, 1985:129.

7 Marsden CD. The problem of adult-onset idiopathic torsion dystonia and other isolated dyskinesias in adult life. Adv Neurol 1976;14:259-76.

8 Mitscherlich M. The theory and therapy of hyperkineses (torticollis). Psychother Psychosom 1979;32:306-12.

9 Choppy-Jacolin M, Ferrey G, Demaria C. A psychometric study of 34 patients afflicted with spasmodic torticollis. Acta Neurol Scand 1977;55:483-92.

10 Matthews WB, Beasley P, Parry-Jones W, et al. Spasmodic torticollis: a combined clinical study. F Neurol Neurosurg Psychiatry 1978;41:485-92.

11 Jahanshahi $M$, Marsden CD. Personality in torticollis: a controlled study. Psychol Med 1988;18:375-87.

12 Rondot P, Marchand MP, Dellatolas G. Spasmodic torticollis: review of 220 patients. Can $\mathcal{f}$ Neurol Sci 1991;18:143-51.

13 Scheidt CE. Psychological distress and psychopathology in spasmodic torticollis: clinical assessment and psychometric spasmodic torticollis: clinical assessment and psychometric
finding. Psychother Psychosom Med Psychol 1995;45:183-91.

14 Patterson RM, Little, SC. Spasmodic torticollis. f Nerv Ment Dis 1943;98:571-99.

15 Paterson MT. Spasmodic torticollis. Results of psychotherapy in 21 cases. Lancet 1945;ii:556-9.

16 Meares A. Features which distinguish groups of spasmodic torticollis. F Psychosom Res 1971;15:1-11.

17 Scheidt CE, Heinen F, Nickel T, et al. Spasmodic torticollis: a multicentre study on behavioural aspects IV: psychopathology. Behav Neurol 1996;9:97-103.

18 Jahanshahi M, Marsden CD. Body concept, disability and depression in patients with spasmodic torticollis. Behav Neurol 1990;3:117-31.

19 Jahanshahi M, Marsden CD. Psychological functioning before and after treatment of torticollis with botulinum toxin. F Neurol Neurosurg Psychiatry 1992;55:229-31.

20 Jahanshahi M, Marsden CD. Depression in torticollis. A Jahanshahi M, Marsden CD. Depression in to
controlled study. Psychol Med 1988;18:925-33.

21 Jahanshahi M. Psychosocial factors and depression in torticollis. f Psychosom Res 1991;35:493-507.

22 Tsui JKC, Eisen AJ, Stoessl AJ, et al. Double-blind study of botulinum toxin in spasmodic torticollis. Lancet 1986;ii: 245-7.

23 Wittchen HU, Wunderlich U, Gruschwitz S, et al. Strukturiertes klinisches Interview für DSM-IV, Achse I (SKID). Göttingen: Hogrefe, 1997.

24 American Psychiatric Association. Diagnostic and statistical manual of mental disorders: 4 th ed. Washington, DC: APA, 1994

25 Williams JBW, Gibbon M, First MB, et al. The structured clinical interview for DSM-III-R (SCID): II. Multisite testretest reliability. Arch Gen Psychiatry 1992;49:630-6.

26 Spitzer RL, Williams JBW, Gibbon M, et al. The structured clinical interview for DSM-III-R (SCID). I. History, rationale, and description. Arch Gen Psychiatry 1992;49: 624-9.

27 Wittchen HU, Essau CA, Zerssen D, et al. Lifetime and 6 month prevalence of mental disorders in the Munich follow up study. Eur Arch Psychiatry Clin Neurosci 1992;241:247$u_{58}$.

28 Derogatis LR. SCL-90-R. Administration, scoring and procedures manual. Baltimore: John Hopkins University, Clinical Psychometrics Research Unit, 1977.

29 Franke G. SCL-90-R. Die Symptom-Checkliste von Derogatis: Deutsche Version. Göttingen: Beltz Test, 1995.

30 Heimberg RG, Mueller GP, Holt CS, et al. Assessment of anxiety in social interaction and being observed by others.: The social interaction anxiety scale and the social phobia scale. Behav Ther 1992;23:53-73.

31 Stangier U, Heidenreich T, Beradi A, et al. Die Erfassung sozialer Phobie durch die social interaction anxiety scale (SIAS) and die Social Phobia Scale (SPS). Z Klin Psychol 1999;28:28-36.

32 Muthny FA. Freiburger Fragebogen zur Krankheitsverarbeitung (FKV). Manual. Weinheim; Beltz, 1989.

33 Maier-Diewald W, Wittchen HU, Hecht H, et al. Die Maier-Diewald W, Wittchen HU, Hecht $\mathrm{H}$, et al. Die
Münchner Ereignisliste (MEL): Anwendungsmanual.
Munich: Max-Planck-Institut für Psychiatrie, 1983 .

34 Burnam MA, Hough RL, Escobar JI, et al. Six-month prevalence of specific psychiatric disorders among Mexican 
Americans and non-hispanic whites in Los Angeles. Arch Gen Psychiatry 1987;44:687-94.

35 Blumer D, Montouris G, Herrmann B. Psychiatric morbidity in seizure patients on a neurodiagnostic monitoring unit. F Neuropsychiatry Clin Neurosci 1995;7:445-56.

36 Guidetti V, Galli F, Fabrizi P, et al. Headache and psychiatric comorbidity. Clinical aspects and outcome in an 8 year follow up study. Cephalalgia 1998;18:455-62.

37 Wenzel T, Schnider P, Wimmer A, et al. Psychiatric comorbidity in patients with spasmodic torticollis. $\mathcal{F}$ Psychosom Res 1998;44:687-90.

38 Stein MB, Walker JR, Forde DR. Setting diagnostic thresholds for social phobia: considerations from a community survey of social anxiety. Am F Psychiatry 1994;151:408-12.
39 Magee WJ, Eaton Ww, Wittchen HU, et al. Agoraphobia, simple phobia, and social phobia in the national comorbidsimple phobia, and social phobia in the national
ity survey. Arch Gen Psychiatry 1996;53:159-68.

40 Stein MB, Heuser IJ, Juncos JL, et al. Anxiety disorders in patients with Parkinson's disease. Am F Psychiatry 1990; 147:217-20.

41 Herschbach P, Henrich G. The significance of objective determinants for the subjective quality of life. Psychosom Med 1998;60:113-14.

42 Waddell G. Low back disability. A syndrome of Western civilization. Neurosurg Clin North Amer 1991;2:719-38.

43 Allison TG, Williams TE, Miller TD, et al. Medical and economic costs of psychologic distress in patients with coronary artery disease. Mayo Clinic Proceedings 1995;70:734-42. 